\title{
MONODROMY GROUPS OF HYPERGEOMETRIC FUNCTIONS SATISFYING ALGEBRAIC EQUATIONS
}

\author{
Mitsuo Kato And Masatoshi Noumi
}

(Received May 11, 2001, revised May 7, 2002)

\begin{abstract}
The solutions of the algebraic equation $y^{m n}+x y^{m p}-1=0$ with $n>p$ and $m \geq 2$ satisfy a generalized hypergeometric differential equation with imprimitive finite irreducible monodromy group. Thanks to this fact, we can determine the monodromy group and the Schwarz map of the differential equation.
\end{abstract}

1. Introduction. A generalized hypergeometric function

$$
{ }_{n} F_{n-1}\left(a_{0}, a_{1}, a_{2}, \ldots, a_{n-1} ; b_{1}, b_{2}, \ldots, b_{n-1} ; z\right)=\sum_{k=0}^{\infty} \frac{\prod_{j=0}^{n-1}\left(a_{j}, k\right)}{\prod_{j=1}^{n-1}\left(b_{j}, k\right) k !} z^{k},
$$

where $(a, k)=\Gamma(a+k) / \Gamma(a)$ satisfies a Fuchsian differential equation

$$
{ }_{n} E_{n-1}\left(a_{0}, a_{1}, a_{2}, \ldots, a_{n-1} ; b_{1}, b_{2}, \ldots, b_{n-1}\right)
$$

of rank $n$ with singularities at $z=0,1$ and $\infty$. Beukers and Heckman [B-H] determined ${ }_{n} E_{n-1}$ with finite irreducible monodromy groups. In [Kt], for ${ }_{3} E_{2}$ with finite irreducible primitive monodromy groups, Schwarz maps of $\boldsymbol{P}^{1}-\{0,1, \infty\}$ to $\boldsymbol{P}^{2}$ defined by linearly independent three solutions are studied. The images of Schwarz maps and their single-valued inverse maps are determined.

1.1. As stated in Theorem 5.8 in [B-H], under some condition, ${ }_{n} E_{n-1}$ with irreducible imprimitive monodromy group is essentially given by

$$
{ }_{n} E_{n-1}\left(\frac{-\alpha}{p}, \frac{-\alpha+1}{p}, \ldots, \frac{-\alpha+p-1}{p}, \frac{\alpha}{q}, \frac{\alpha+1}{q}, \ldots, \frac{\alpha+q-1}{q} ; \frac{1}{n}, \ldots, \frac{n-1}{n}\right),
$$

where $(p, q)=1$ and $n=p+q$.

If we put $z=(-p)^{p} q^{q} n^{-n} x^{n}$, the generalized binomial function (see Section 2)

$$
\psi(\alpha,-p / n, x)
$$

2000 Mathematics Subject Classification. Primary 33C20.

Key words and phrases. Hypergeometric function, generalized binomial function, monodromy group. 
is (as a multi-valued function of $z$ ) a solution of (1.1). We remark that (1.2) is a typical example of quasi-hypergeometric function studied in [A-I]. If $\alpha=-1 /(m n)$ with $m \geq 1$, then (1.2) is also a solution of the algebraic equation

$$
y^{m n}+x y^{m p}-1=0 .
$$

These facts were found by Lambert (see [Brn, p. 307]), Mellin (see [Blr]) and others.

Let $\alpha=-1 /(m n)$ with $m \geq 2$. Then a set of linearly independent $n$ solutions of (1.3) form a fundamental system of solutions of (1.1). As a consequence, we have the following results. The projective monodromy group of (1.1) is imprimitive and irreducible of order $m^{n-1} n$ ! (Corollary 4.6). The closure of the image of the Schwarz map of (1.1) defined by the ratio of linearly independent $n$ solutions is an irreducible algebraic curve projectively isomorphic to

$$
\left\{\left[y_{0}: y_{1}: \cdots: y_{n-1}\right] \in \boldsymbol{P}^{n-1} \mid \sigma_{k}\left(y_{0}^{m}, y_{1}^{m}, \ldots, y_{n-1}^{m}\right)=0,1 \leq k \leq n-1, k \neq n-p\right\},
$$

where $\sigma_{k}$ is the elementary symmetric function of degree $k$ (Theorem 4.5).

1.2. As applications, we state several topics for $n=3$ case in Section 5. We give a proof of Cardano's formula for a cubic equation, using properties of generalized binomial functions. We also give a uniformization of ${ }_{3} E_{2}$ by theta functions, that is, if we put $z=J(\tau)$, the elliptic modular function, then the solutions of (1.1) with $\alpha=-1 / 12, p=1, q=2$ turn out to be single-valued functions of $\tau$ and are expressed by the zero values of theta functions.

2. Generalized binomial function. In this section, we summarize several known results which can be found in [Brn], [Blr], etc.

For any complex numbers $\alpha$ and $s$, put

$$
\begin{aligned}
& c_{0}(\alpha, s)=1, \\
& c_{k}(\alpha, s)=\alpha(\alpha+k s+1, k-1) / k ! \quad(k \geq 1),
\end{aligned}
$$

and define

$$
\psi(\alpha, s, x)=\sum_{k=0}^{\infty} c_{k}(\alpha, s) x^{k}
$$

We call $\psi(\alpha, s, x)$ a generalized binomial function because $\psi(\alpha, 0, x)=(1-x)^{-\alpha}$.

We will prove some properties of $\psi(\alpha, s, x)$.

LEMMA 2.1.

$$
\psi(\alpha, s, x)=\psi(-\alpha,-s-1,-x)
$$

PROOF.

$$
\begin{aligned}
(-1)^{k} & c_{k}(-\alpha,-s-1) \\
& =(-1)^{k}(-\alpha)(-\alpha-(s+1) k+1, k-1) / k ! \\
& =\alpha(\alpha+s k+k-1)(\alpha+s k+k-2) \cdots(\alpha+s k+1) \\
& =c_{k}(\alpha, s) .
\end{aligned}
$$


We note that $\psi(\alpha,-1, x)=(1+x)^{\alpha}$ and $\psi(0, s, x)=1$.

PROPOSITION 2.2. If none of $\alpha, s, s+1$ is zero, then the radius of convergence of $\psi(\alpha, s, x)$ is $\left|s^{s} /(s+1)^{s+1}\right|$, where $z^{z}$ denotes the principal value.

Proof. Put

$$
\tilde{c}_{k}(\alpha, s)=(\alpha+s k+1, k-1) / k !=\frac{\Gamma(\alpha+(s+1) k)}{\Gamma(1+k) \Gamma(\alpha+1+s k)} .
$$

Then the radius of convergence of $\psi(\alpha, s, x)$ is the reciprocal of the upper limit of $\left|\tilde{c}_{k}\right|^{1 / k}$.

First assume that $s$ is not a negative real number. Then, from the Stirling's formula:

$$
\Gamma(z) \sim \sqrt{2 \pi} z^{z-1 / 2} e^{-z} \quad \text { as } \quad z \rightarrow \infty \quad \text { and } \quad|\arg z|<\pi-\delta, \quad \delta>0,
$$

we have

$$
\begin{aligned}
\left|\tilde{c}_{k}(\alpha, s)\right|^{1 / k} & \sim \frac{\left|(\alpha+(s+1) k)^{s+1}\right|}{(1+k)\left|(\alpha+1+s k)^{s}\right|} \sim\left|\frac{\alpha+(s+1) k}{1+k}\left(\frac{\alpha+(s+1) k}{\alpha+1+s k}\right)^{s}\right| \\
& \sim\left|(s+1)^{s+1} / s^{s}\right| .
\end{aligned}
$$

This proves the proposition for $s$ which is not a negative real number.

Assume $-1<s<0$. For large $k \in N$, choose $n_{k} \in N$ and $\delta_{k}$ with $0 \leq \delta_{k}<1$ such that

$$
\operatorname{Re}(\alpha)+s k=-n_{k}-\delta_{k} .
$$

Then

$$
\begin{aligned}
\left|\tilde{c}_{k}(\alpha, s)\right|= & |(\alpha+1+s k, k-1)| / k ! \\
= & \left|(\alpha+1+s k) \cdots\left(\alpha+1+s k+n_{k}-1\right)\right| \\
& \times\left|\left(\alpha+1+s k+n_{k}\right) \cdots(\alpha+(s+1) k-1)\right| / k ! \\
= & \left|\left(-\alpha-s k-n_{k}, n_{k}\right)\right| \cdot\left|\left(\alpha+s k+n_{k}+1, k-1-n_{k}\right)\right| / k ! \\
= & \frac{|\Gamma(-\alpha-s k)| \cdot|\Gamma(\alpha+(s+1) k)|}{\left|\Gamma(1+k) \Gamma\left(-\alpha-s k-n_{k}\right) \Gamma\left(\alpha+s k+n_{k}+1\right)\right|} .
\end{aligned}
$$

If $s$ is a rational number, then the set $\delta:=\left\{\delta_{k} \mid k \in N\right\}$ is finite, otherwise $\delta$ is dense in the open interval $(0,1)$. In any case we have

$$
\begin{aligned}
\limsup _{k \rightarrow \infty}\left|\tilde{c}_{k}(\alpha, s)\right|^{1 / k} & =\lim _{k \rightarrow \infty}\left|\frac{(-\alpha-s k)^{-s}(\alpha+(s+1) k)^{s+1}}{1+k}\right| \\
& =\lim _{k \rightarrow \infty}\left|\left(\frac{-\alpha-s k}{1+k}\right)^{-s}\left(\frac{\alpha+(s+1) k}{1+k}\right)^{s+1}\right| \\
& =\left|(-s)^{-s}(s+1)^{s+1}\right|=\left|(s+1)^{s+1} / s^{s}\right| .
\end{aligned}
$$

This proves the proposition for $s$ with $-1<s<0$. From Lemma 2.1, the proposition holds for any negative real number $s$ which is not -1 . This completes the proof.

LEMMA 2.3 .

$$
c_{k}(\alpha, s)-c_{k}(\alpha-1, s)=c_{k-1}(\alpha+s, s), \quad k \geq 1 .
$$


PROOF.

$$
\begin{aligned}
c_{k}(\alpha, s) & -c_{k}(\alpha-1, s) \\
= & \frac{\alpha(\alpha+k s+1, k-1)-(\alpha-1)(\alpha+k s, k-1)}{k !} \\
= & \frac{(\alpha+s)(\alpha+s+(k-1) s+1, k-2)}{(k-1) !}=c_{k-1}(\alpha+s, s) .
\end{aligned}
$$

PROPOSITION 2.4. We have the following two equalities.

$$
\begin{aligned}
& \psi(\alpha, s, x)-\psi(\alpha-1, s, x)=x \psi(\alpha+s, s, x), \\
& \psi(\alpha+\beta, s, x)=\psi(\alpha, s, x) \psi(\beta, s, x) .
\end{aligned}
$$

PROOF. (2.5) follows immediately from (2.4).

Proof of (2.6). It is sufficient to prove

$$
c_{k}(\alpha+\beta, s)=\sum_{i+j=k} c_{i}(\alpha, s) c_{j}(\beta, s),
$$

which is proved by induction for $k$. Consider

$$
d_{k}(\beta)=c_{k}(\alpha+\beta, s)-\sum_{i+j=k} c_{i}(\alpha, s) c_{j}(\beta, s)
$$

as a polynomial of $\beta$ ( $\alpha$ being a parameter) of degree at most $k$. From (2.4), we have

$$
d_{k}(\beta)-d_{k}(\beta-1)=d_{k-1}(\beta+s),
$$

which vanishes by induction. Hence $d_{k}(\beta)$ must be constant $C$. Since $c_{j}(0, s)=0$ for $j>0$, we hace $C=d_{k}(0)=0$. This completes the proof of (2.7) whence of (2.6).

COROllary 2.5. Let $\psi^{\prime}(s, x)=\partial \psi / \partial \alpha(0, s, x)$. Then we have the following:

(1) $\psi^{\prime}(s, x)$ is holomorphic in $\left\{x|| x|<| s^{s} /(s+1)^{s+1} \mid\right\}$ with $\psi^{\prime}(s, 0)=0$.

(2) $\psi(\alpha, s, x)=\exp \left(\alpha \psi^{\prime}(s, x)\right)$.

PROOF. (1) holds because $\psi^{\prime}(s, x)=\sum_{k \geq 1} \tilde{c}_{k}(\alpha, s) x^{k}$, where $\tilde{c}_{k}(\alpha, s)=c_{k}(\alpha, s) / \alpha$ as in the proof of Proposition 2.2. (2) follows from (2.6).

Proposition 2.6. Let $\varepsilon_{k}=e^{2 \pi i / k}$. For positive integers $p, q$ with $n=p+q$, the equation (1.3) with $m=1$

$$
y^{n}+x y^{p}-1=0
$$

has solutions

$$
f_{j}(x):=\varepsilon_{n}^{j} \psi\left(-1 / n,-p / n, \varepsilon_{n}^{p j} x\right), \quad 0 \leq j \leq n-1,
$$

in a neighborhood of $x=0$,

$$
\begin{aligned}
& \varepsilon_{p}^{-j} x^{-1 / p} \psi\left(1 / p, q / p,-\left(\varepsilon_{p}^{-j} x^{-1 / p}\right)^{n}\right), \quad 0 \leq j \leq p-1, \\
& \varepsilon_{q}^{j}(-x)^{1 / q} \psi\left(-1 / q, p / q,-\left(\varepsilon_{q}^{j}(-x)^{1 / q}\right)^{-n}\right), \quad 0 \leq j \leq q-1,
\end{aligned}
$$


in a neighborhood of $x=\infty$.

ProOF. Put $s=-p / n$ and $\alpha=0$ in (2.5). Then we have

$$
1-\psi(-1, s, x)=x \psi(-p / n, s, x),
$$

which is equivalent to

$$
\psi(-1 / n, s, x)^{n}+x \psi(-1 / n, s, x)^{p}-1=0 .
$$

If we replace $x$ by $\varepsilon_{n}^{p j} x$, we know that (2.9) are solutions of (2.8).

Put $s=q / p$ and $\alpha=1$ in (2.5). Then we have

$$
\psi(1 / p, s, x)^{p}-1=x \psi(1 / p, s, x)^{n},
$$

which is equivalent to

$$
\left[(-x)^{1 / n} \psi(1 / p, s, x)\right]^{n}+(-x)^{-p / n}\left[(-x)^{1 / n} \psi(1 / p, s, x)\right]^{p}-1=0 .
$$

Put $x_{1}=(-x)^{-p / n}$, and write $x$ instead of $x_{1}$. Then we know that functions in (2.10) are solutions of (2.8).

Now, put $s=p / q$ and $\alpha=-s$ in (2.5). Then we have

$$
\psi(-1 / q, s, x)^{n}-\psi(-1 / q, s, x)^{p}+x=0 .
$$

Then, by the same way as above, we know that functions in (2.11) are solutions of (2.8). This completes the proof.

COROLLARY 2.7. If $\sigma_{k}\left(y_{0}, y_{1}, \ldots, y_{n-1}\right)$ denotes the elementary symmetric function of degree $k$, then we have

$$
\begin{aligned}
\sigma_{k}\left(f_{0}(x), f_{1}(x), \ldots, f_{n-1}(x)\right) & =0, \quad 1 \leq k \leq n-2, k \neq n-p, \\
\sigma_{n-p}\left(f_{0}(x), f_{1}(x), \ldots, f_{n-1}(x)\right) & =(-1)^{n-p} x, \\
\sigma_{n}\left(f_{0}(x), f_{1}(x), \ldots, f_{n-1}(x)\right) & =(-1)^{n-1} .
\end{aligned}
$$

For any positive integer $n$, put

$$
\varphi_{j}(\alpha, s, x)=x^{j} \sum_{l=0}^{\infty} c_{j+l n}(\alpha, s) x^{l n} .
$$

Then we have

$$
\psi(\alpha, s, x)=\sum_{j=0}^{n-1} \varphi_{j}(\alpha, s, x) .
$$


PROPOSITION 2.8. Let $s=-p / n$ and $n=p+q$. Then we have

$$
\begin{aligned}
& \varphi_{j}(\alpha, s, x)=c_{j}(\alpha, s) x^{j} \\
& \times{ }_{n} F_{n-1}\left(\frac{-\alpha+\mu}{p}+\frac{j}{n}, 0 \leq \mu \leq p-1, \frac{\alpha+v}{q}+\frac{j}{n}, 0 \leq v \leq q-1 ;\right. \\
& \left.\quad \frac{j+1}{n}, \ldots, \frac{n-1}{n}, \frac{n+1}{n}, \ldots, \frac{n+j}{n} ; \frac{(-1)^{p} p^{p} q^{q}}{n^{n}} x^{n}\right) .
\end{aligned}
$$

PROOF. If $k=n l(l \geq 1)$, then we have

$$
\begin{aligned}
& c_{k}(\alpha, s)= \frac{1}{k !} \alpha(\alpha-p l+1, n l-1)=\frac{1}{k !} \alpha(\alpha-p l+1, p l-1)(\alpha, q l) \\
&=(-1)^{p l} \frac{(-\alpha, p l)(\alpha, q l)}{(1, n l)} \\
&= p^{p l} q^{q l} \prod_{\mu=0}^{p-1}(-\alpha / p+\mu / p, l) \prod_{\nu=0}^{q-1}(\alpha / q+v / q, l) \\
& n^{n l} \prod_{\lambda=0}^{n-1}(1 / n+\lambda / n, l)
\end{aligned}
$$

If $k=n l+j(1 \leq j \leq n-1)$, then we have

$$
\begin{aligned}
& c_{k}(\alpha, s) \\
& \quad=\frac{1}{k !} \alpha\left(\alpha-\frac{p}{n}(n l+j)+1, n l+j-1\right) \\
& =\frac{1}{j !(j+1, n l)} \alpha\left(\alpha-\frac{p}{n}(n l+j)+1, p l\right)\left(\alpha-\frac{p j}{n}+1, j-1\right)\left(\alpha+\frac{q j}{n}, q l\right) \\
& =\frac{\alpha(\alpha+q j / n-j+1, j-1)}{j !}(-1)^{p l} \frac{(-\alpha+p j / n, p l)(\alpha+q j / n, q l)}{(j+1, n l)} \\
& =c_{j}(\alpha, s)(-1)^{p l} \frac{p^{p l} q^{q l} \prod_{\mu=0}^{p-1}(-\alpha / p+j / n+\mu / p, l) \prod_{\nu=0}^{q-1}(\alpha / q+j / n+v / q, l)}{n^{n l} \prod_{\lambda=0}^{n-1}((j+1) / n+\lambda / n, l)}
\end{aligned}
$$

This implies (2.18).

COROLlaRY 2.9. Let $s=-p / n, n=p+q$ and $\varepsilon_{n}=e^{2 \pi i / n}$. Then $\psi\left(\alpha, s, \varepsilon_{n}^{k} x\right)$ is, as a multi-valued function of $z=(-p)^{p} q^{q} n^{-n} x^{n}$, a solution of the differential equation (1.1). If $c_{j}(\alpha, s) \neq 0$ for $0 \leq j \leq n-1$, then $\psi\left(\alpha, s, \varepsilon_{n}^{k} x\right) \quad 0 \leq k \leq n-1$ are linearly independent. 
Proof. From (2.18), we know that $\varphi_{j}(\alpha, s, x)$ is a solution of (1.1) (see the lemma below). From (2.16) and (2.17), we have

$$
\psi\left(\alpha, s, \varepsilon_{n}^{k} x\right)=\sum_{j=0}^{n-1} \varepsilon_{n}^{j k} \varphi_{j}(\alpha, s, x),
$$

which is thus a solution of (1.1). If $c_{j}(\alpha, s) \neq 0$, then $\varphi_{j}(\alpha, s, x) \neq 0$ and $\psi\left(\alpha, s, \varepsilon_{n}^{k} x\right)$, $0 \leq k \leq n-1$, are linearly independent from (2.19).

The following lemma is well-known.

LEMMA 2.10. If $b_{0}=1$, then the differential equation

$$
{ }_{n} E_{n-1}\left(a_{0}, a_{1}, a_{2}, \ldots, a_{n-1} ; b_{1}, b_{2}, \ldots, b_{n-1}\right)
$$

has solutions

$$
\begin{aligned}
& z^{1-b_{j}} F_{n-1}\left(a_{0}+1-b_{j}, \ldots, a_{n-1}+1-b_{j} ;\right. \\
& \left.\quad b_{0}+1-b_{j}, \ldots, b_{j} \widehat{+1-} b_{j}, \ldots, b_{n-1}+1-b_{j} ; z\right) ; 0 \leq j \leq n-1
\end{aligned}
$$

at $z=0$ and

$$
\begin{aligned}
& z^{-a_{j}}{ }_{n} F_{n-1}\left(a_{j}+1-b_{0}, \ldots, a_{j}+1-b_{n-1} ;\right. \\
& \left.\quad a_{j}+1-a_{0}, \ldots, a_{j} \widehat{+1-} a_{j}, \ldots, a_{j}+1-a_{n-1} ; 1 / z\right) ; 0 \leq j \leq n-1
\end{aligned}
$$

at $z=\infty$.

PROOF. ${ }_{n} E_{n-1}$ is defined by

$$
\left[\prod_{j=0}^{n-1}\left(\vartheta+b_{j}-1\right)-z \prod_{j=0}^{n-1}\left(\vartheta+a_{j}\right)\right] u=0,
$$

where $\vartheta=z \partial / \partial z$ (see [Bly]). It is easily verified that functions in Lemma satisfy (2.20).

REMARK 2.1. If $s=p / q$ with $n=p+q$, then we have, for $0 \leq j \leq q-1$,

$$
\begin{aligned}
& \varphi_{j}(\alpha, s, x)=x^{j} \sum_{l=0}^{\infty} c_{j+l q}(\alpha, s) x^{l q} \\
& =c_{j}(\alpha, s) x^{j}{ }_{n} F_{n-1}\left(\frac{\alpha}{n}+\frac{j}{q}, \frac{\alpha+1}{n}+\frac{j}{q}, \ldots, \frac{\alpha+n-1}{n}+\frac{j}{q}\right. \\
& \left.\quad \frac{\alpha+1}{p}+\frac{j}{q}, \ldots, \frac{\alpha+p}{p}+\frac{j}{q}, \frac{1+j}{q}, \ldots, \frac{q-1}{q}, \frac{q+1}{q}, \ldots, \frac{q+j}{q} ; \frac{n^{n}}{p^{p} q^{q}} x^{q}\right) .
\end{aligned}
$$

3. Global properties of solutions of $y^{n}+x y^{p}-1=0$. Assume $s(s+1) \neq 0$. Put $\Delta(s)=\left\{x|| x|<| s^{s} /(s+1)^{s+1} \mid\right\}$. Then $\psi(\alpha, s, x)$ and $\psi^{\prime}(s, x)=\partial \psi / \partial \alpha(0, s, x)$ are holomorphic in $\Delta(s)$ (Proposition 2.2 and Corollary 2.5). 
Lemma 3.1. Assume $s \in \boldsymbol{R}$. Then we have $|\arg \psi(-1, s, x)|<\pi$, or equivalently, $\left|\operatorname{Im} \psi^{\prime}(s, x)\right|<\pi$ in $\Delta(s)$.

Proof. Assume $\left|\operatorname{Im} \psi^{\prime}\left(s, x_{1}\right)\right|=\pi$ for some $x_{1} \in \Delta(s)$. From (2.5) and (2) of Corollary 2.5 , we have

$$
\exp \left(-s \psi^{\prime}\left(s, x_{1}\right)\right)\left(1-\exp \left(-\psi^{\prime}\left(s, x_{1}\right)\right)\right)=x_{1} \text {. }
$$

This implies $\theta:=\arg x_{1}=( \pm s+2 n) \pi$ for some $n \in Z$. Since $\operatorname{Im} \psi^{\prime}(s, 0)=0$, there exist a positive number $t_{0}\left(\leq\left|x_{1}\right|\right)$ such that

$$
\left|\operatorname{Im} \psi^{\prime}\left(s, t e^{i \theta}\right)\right|<\pi \text { for } 0<t<t_{0} \text { and }\left|\operatorname{Im} \psi^{\prime}\left(s, t_{0} e^{i \theta}\right)\right|=\pi .
$$

Put $x_{0}=t_{0} e^{i \theta}$ and $b_{0}=\psi\left(-1, s, x_{0}\right)(<0)$. Since $y=\psi(-1, s, x)$ defines an open map, $\psi\left(-1, s, e^{i \theta} t\right)$ maps some open interval $\left(t_{0}-\delta, t_{0}+\delta\right)$ onto some open interval $\left(b_{0}-\delta^{\prime}, b_{0}+\right.$ $\left.\delta^{\prime \prime}\right)$. This contradicts the choice of $t_{0}$.

We assume $(p, q)=1$ and put $n=p+q$. Recall that $f_{j}(x), 0 \leq j \leq n-1$ given by (2.9) are the solutions of the equation (2.8). The equation (2.8) has multiple roots at

$$
x_{j}:=e\left(\frac{-p(1+2 j)}{2 n}\right)(p / n)^{-p / n}(q / n)^{-q / n}, \quad 0 \leq j \leq n-1,
$$

where $e(x)=e^{2 \pi i x}$ and at $x=\infty$. Note that $x=x_{j}$ are solutions of

$$
(-p)^{p} q^{q} n^{-n} x^{n}=1 .
$$

LeMma 3.2. At $x=x_{j}$, the equation (2.8) has a double root

$$
e((1+2 j) / 2 n)(p / q)^{1 / n}
$$

and $n-2$ simple roots.

Proof. The double root of the equation (2.8) is uniquely determined by (2.8) and $n y^{n-1}+p x y^{p-1}=0$.

We know that $f_{j}(x)$ are holomorphic in $\Delta:=\Delta(-p / n)=\left\{x|| x \mid<(p / n)^{-p / n}(q / n)^{-q / n}\right\}$ and continuous in the closure $\bar{\Delta}$ of $\Delta$.

Put

$$
D_{j}=f_{j}(\bar{\Delta}) .
$$

Then we have $D_{j}=e(j / n) D_{0}$ and put $D_{n}=D_{0}$.

LEMMA 3.3 .

$$
\begin{aligned}
& \left(\frac{-1+2 j}{n}\right) \pi \leq \arg y \leq\left(\frac{1+2 j}{n}\right) \pi \quad \text { for } y \in D_{j}, \\
& D_{j} \cap D_{j+1}=\left\{f_{j}\left(x_{j}\right)\right\}=\left\{f_{j+1}\left(x_{j}\right)\right\}=\left\{e((1+2 j) / 2 n)(p / q)^{1 / n}\right\},
\end{aligned}
$$

and $D_{j} \cap D_{k}=\emptyset$ if $j-k \neq \pm 1$. 
Proof. The inequalities (3.4) follow from Lemma 3.1 and (2) of Corollary 2.5. These inequalities imply that $D_{j} \cap D_{k}=\emptyset$ if $j-k \neq \pm 1$. Since any element of $D_{j} \cap D_{j+1}$ is one of (3.2), we have

$$
D_{j} \cap D_{j+1}=\left\{e((1+2 j) / 2 n)(p / q)^{1 / n}\right\}
$$

from (3.4). From Lemma 3.2, (3.5) follows.

COROLLARY 3.4. Let $\gamma_{0}$ be a loop starting and ending at the origin and once surrounding $x_{0}$. Let $\gamma_{j}=e(-p j / n) \gamma_{0}$. Then, by the analytic continuation along $\gamma_{j}, f_{j}(x)$ and $f_{j+1}(x)$ are interchanged and other $f_{k}(x)$ are unchanged.

Proof. Assume $\gamma_{0}$ (hence any $\gamma_{j}$ ) acts trivially on $\left\{f_{0}, \ldots, f_{n-1}\right\}$. Then $f_{j}(x)$ are entire functions. This contradicts Proposition 2.6.

Definition 3.1. Let $E$ be a Fuchsian linear differential equation of rank $n$ on $\boldsymbol{P}^{1}$. Let $Z=\boldsymbol{P}^{1}-\{$ singular points of $E\}$. Fix a base point $z_{b} \in Z$, and let $V$ be the set of germs of holomorphic solutions of $E$ at $z_{b}$. For any $\gamma \in \pi_{1}\left(Z, z_{b}\right)$ and $f \in V$, the analytic continuation $\gamma_{*} f$ of $f$ along $\gamma$ is again in $V$. We consider $\gamma_{*}$ an element of $G L(V)$ and call the set $M(E)$ of all $\gamma_{*}$ the monodromy group of $E$ and $M(E) /$ (its center) the projective monodromy group of $E$.

We say that $M(E)$ is (or $E$ is) reducible if there exists a non trivial subspace $V_{1}$ of $V$ which is invariant under the action of $M(E)$ and say $M(E)$ is (or $E$ is) irreducible if $M(E)$ is not reducible.

We say that $M(E)$ is (or $E$ is) imprimitive if $V$ has a direct sum decomposition $V=$ $V_{1}+V_{2}+\cdots+V_{k}$ such that any element of $M(E)$ induces a permutation of $\left\{V_{1}, V_{2}, \ldots, V_{k}\right\}$.

Choose a basis $v_{j}(z), 1 \leq j \leq n$ of $V$. Then we have a holomorphic map

$$
v(z)=\left[v_{1}(z): v_{2}(z): \cdots: v_{n}(z)\right]
$$

of a neighbourhood of $z_{b}$ into $\boldsymbol{P}^{n-1}$. By taking analytic continuations of $v$, we have a multivalued map (again denoted by) $v$ of $Z$ into $\boldsymbol{P}^{n-1}$ which we call a Schwarz map of $E$.

Remark 3.1. If the Schwarz map has a single-valued inverse map $\pi_{E}$, then the projective monodromy group of $E$ is isomorphic to the covering transformation group of $\pi_{E}$.

The map of $\Delta$ to $\boldsymbol{P}^{n-1}$ defined by $\left[f_{0}(x): f_{1}(x): \cdots: f_{n-1}(x)\right]$ is extended to a multivalued map of $\boldsymbol{C}-\left\{x_{0}, \ldots, x_{n-1}\right\}$ to $\boldsymbol{P}^{n-1}$ by the analytic continuations. Take the closure of its image in $\boldsymbol{P}^{n-1}$, which we denote by $X_{n, p}$.

Proposition 3.5. Let $\sigma_{k}(y)=\sigma_{k}\left(y_{0}, y_{1}, \ldots, y_{n-1}\right)$ be the elementary symmetric function of degree $k$. Then we have the equality

$$
X_{n, p}=\left\{\left[y_{0}: y_{1}: \cdots: y_{n-1}\right] \in \boldsymbol{P}^{n-1} \mid \sigma_{k}(y)=0,1 \leq k \leq n-1, k \neq q\right\} .
$$

Put

$$
\pi_{n, p}\left(\left[y_{0}: y_{1}: \cdots: y_{n-1}\right]\right)=(-1)^{n} \frac{p^{p} q^{q}}{n^{n}} \frac{\left(\sigma_{q}\left(y_{0}, \ldots, y_{n-1}\right)\right)^{n}}{\left(\sigma_{n}\left(y_{0}, \ldots, y_{n-1}\right)\right)^{q}} .
$$


Then $z=\pi_{n, p}(y)$ defines an $n !: 1$ rational map of $X_{n, p}$ to $\boldsymbol{P}^{1}$ satisfying

$$
\pi_{n, p}\left(\left[f_{0}(x): f_{1}(x): \cdots: f_{n-1}(x)\right]\right)=(-p)^{p} q^{q} n^{-n} x^{n} .
$$

The branch points of this map are $z=0,1, \infty$ with the ramification indices $n, 2, p q$, respectively. The covering transformation group is isomorphic to the symmetric group $S_{n}$ of order $n !$

Proof. Denote by $\hat{X}_{n, p}$ the set of common zeros of $\sigma_{k}, 0 \leq k \leq n-2, k \neq q$. From Bezout's theorem, $\left.\pi_{n, p}\right|_{\hat{X}_{n, p}}$ is an $n !: 1$ map of $\hat{X}_{n, p}$ to $\boldsymbol{P}^{1}$. From Corollary 2.7, we have $X_{n, p} \subset \hat{X}_{n, p}$, that is, $X_{n, p}$ is an irreducible component of $\hat{X}_{n, p}$. From Corollary 2.7, (3.8) holds and from Corollary 3.4, we know that $S_{n}$ acts on each fiber of $\left.\pi_{n, p}\right|_{X_{n, p}}$. Consequently, we must have $\hat{X}_{n, p}=X_{n, p}$.

The equality (3.8) implies that the ramification index is $n$ at $z=0$. From Corollary 3.4, the index at $z=1$ is 2 . From Proposition 2.6, we know that the ramification index at $z=\infty$ is $p q$. This completes the proof.

The statement (2) of the following corollary is proved in [B-H, Proposition 2.6].

COROLlary 3.6. (1) If $p<n-1$, then $\psi\left(-1 / n,-p / n, \varepsilon_{n}^{k} x\right), 0 \leq k \leq n-1$, are solutions of a differential equation ${ }_{n-1} E_{n-2}$, the projective monodromy group of which is isomorphic to the symmetric group $S_{n}$ of order $n$ !. Any $n-1$ of the above solutions are linearly independent.

(2) The projective monodromy group of

$$
{ }_{n-1} E_{n-2}\left(\frac{1}{n}, \frac{2}{n}, \ldots, \frac{n-1}{n} ; \frac{1}{p}, \ldots, \frac{p-1}{p}, \frac{1}{q}, \ldots, \frac{q-1}{q}\right)
$$

is isomorphic to $S_{n}$.

Proof. Proof of (1). Assume $p<n-1$ or equivalently $q>1$. Put $\alpha=-1 / n$ and $s=-p / n$. Let $q^{*}$ be the integer such that

$$
1 \leq q^{*} \leq n-1 \text { and } q q^{*} \equiv 1 \bmod n .
$$

Then $p^{*}:=n-q^{*}$ also satisfies $p p^{*} \equiv 1 \bmod n$. For $k=p$ or $q$, put $d_{k}=\left(k k^{*}-1\right) / n$. Note $q^{*}>1$ and $d_{q}>0$ because $q>1$. We easily have $c_{q^{*}}(\alpha, s)=0$, and hence $\varphi_{q^{*}}(\alpha, s, x)=0$ (see Proposition 2.8). Since

$$
\left(-\alpha+d_{p}\right) / p=\left(\alpha+q-d_{q}\right) / q=p^{*} / n,
$$

we have

$$
\begin{aligned}
& \varphi_{0}(\alpha, s, x) \\
& ={ }_{n-1} F_{n-2}\left(\frac{-\alpha}{p}, \ldots, \frac{-\alpha+p-1}{p}, \frac{\alpha}{q}, \ldots, \frac{\alpha \widehat{+q-} d_{q}}{q}, \ldots, \frac{\alpha+q-1}{q} ;\right. \\
& \left.\quad \frac{n-1}{n}, \ldots, \frac{\widehat{p^{*}}}{n}, \ldots, \frac{1}{n} ; z\right),
\end{aligned}
$$


where $z=(-1)^{p} p^{p} q^{q} n^{-n} x^{n}$ as before. By the same way, we know that $\left\{\varphi_{j} \mid 0 \leq j \leq n-1, j \neq q^{*}\right\}$ forms a system of fundamental solutions of

$$
\begin{gathered}
{ }_{n-1} E_{n-2}\left(\frac{-\alpha}{p}, \ldots, \frac{-\alpha+p-1}{p}, \frac{\alpha}{q}, \ldots, \frac{\alpha+\widehat{+q} d_{q}}{q}, \ldots, \frac{\alpha+q-1}{q} ;\right. \\
\left.\frac{n-1}{n}, \ldots, \frac{\widehat{p^{*}}}{n}, \ldots, \frac{1}{n}\right) .
\end{gathered}
$$

The equalities (2.19) imply that $\psi\left(-1 / n,-p / n, \varepsilon_{n}^{k} x\right), 0 \leq k \leq n-1$, are solutions of (3.10) and moreover any $n-1$ of them are linearly independent. Since the projective monodromy group of (3.10) is isomorphic to the covering transformation group of $\pi_{n, p}$, which is isomorphic to $S_{n}$ from Proposition 3.5. This completes the proof of (1).

Proof of (2). In (3.9), $p$ and $q$ are symmetric so that we can remain the assumption of $p<n-1$. Put $r=\left(-\alpha+d_{p}\right) / p=\left(\alpha+q-d_{q}\right) / q=p^{*} / n$. Then, from Lemma 2.10, the equation (3.10) has the special solution

$$
\begin{aligned}
& z_{n-1}^{-r} F_{n-2}\left(r, r+\frac{1}{n}, \ldots, r+\frac{q^{*}}{n}, \ldots, r+\frac{n-1}{n} ; 1+\frac{d_{p}}{p}, \ldots, 1+\frac{1}{p},\right. \\
& \left.\quad \frac{p-1}{p}, \ldots, \frac{1+d_{p}}{p}, 1+\frac{q-d_{q}}{q}, \ldots, 1+\frac{1}{q}, \frac{q-1}{q}, \ldots, \frac{q-d_{q}-1}{q} ; 1 / z\right) .
\end{aligned}
$$

Thus the projective monodromy groups of (3.9) and (3.10) are mutually isomorphic, proving (2). This completes the proof.

4. Schwarz map of a family of imprimitive ${ }_{n} E_{n-1}$. Assume $(p, q)=1$ and put

$$
n=p+q, \quad s=-p / n, \quad z=(-p)^{p} q^{q} n^{-n} x^{n}, \quad \varepsilon_{k}=e(1 / k)=e^{2 \pi i / k} .
$$

For an integer $m \geq 2$, put $\alpha=-1 /(m n)$ and define

$$
f_{j}^{(1 / m)}(x)=\varepsilon_{m n}^{j} \psi\left(\alpha, s, \varepsilon_{n}^{p j} x\right) \quad 0 \leq j \leq n-1,
$$

which is a $m$-th root of $f_{j}(x)$. The following lemma is an immediate consequence of the definition (4.1) of $f_{j}^{(1 / m)}$.

LEMMA 4.1. We have

$$
\begin{aligned}
& f_{j}^{(1 / m)}(e(p / n) x)=e(-1 /(m n)) f_{j+1}^{(1 / m)}(x), \quad \text { for } 0 \leq j \leq n-2, \\
& f_{n-1}^{(1 / m)}(e(p / n) x)=e((n-1) /(m n)) f_{0}^{(1 / m)}(x) .
\end{aligned}
$$

When we consider $f_{j}^{(1 / m)}(x)$ as a multi-valued function of $z$, we denote it by $f_{j}^{(1 / m)}(z)$.

LEMMA 4.2. $f_{j}^{(1 / m)}(z), \quad 0 \leq j \leq n-1$, are linearly independent solutions of differential equation (1.1).

Proof. Since $c_{j}(\alpha, s) \neq 0$, for $0 \leq j \leq n-1$, Corollary 2.9 proves the lemma. 
Similar to (3.3), we put

$$
D_{j}^{(1 / m)}=f_{j}^{(1 / m)}(\bar{\Delta}) .
$$

Then we have $D_{j}^{(1 / m)}=e(j /(m n)) D_{0}^{(1 / m)}$ and can prove the following lemma and its corollary from Lemma 3.3 and Corollary 3.4.

LEMMA 4.3.

$$
\begin{aligned}
D_{j}^{(1 / m)} \cap D_{j+1}^{(1 / m)} & =\left\{f_{j}^{(1 / m)}\left(x_{j}\right)\right\}=\left\{f_{j+1}^{(1 / m)}\left(x_{j}\right)\right\} \\
& =\left\{e((1+2 j) /(2 m n))(p / q)^{1 / n}\right\}, 0 \leq j \leq n-2, \\
D_{n-1}^{(1 / m)} \cap e(1 / m) D_{0}^{(1 / m)} & =\left\{f_{n-1}^{(1 / m)}\left(x_{n-1}\right)\right\}=\left\{e(1 / m) f_{0}^{(1 / m)}\left(x_{n-1}\right)\right\} \\
& =\left\{e((2 n-1) /(2 m n))(p / q)^{1 / n}\right\} .
\end{aligned}
$$

Corollary 4.4. Let $\gamma_{j}$ be the loop defined in Corollary 3.4. For $0 \leq j \leq n-2$, by the analytic continuation along $\gamma_{j}, f_{j}^{(1 / m)}(x)$ and $f_{j+1}^{(1 / m)}(x)$ are interchanged and other $f_{k}^{(1 / m)}(x)$ are unchanged; by that along $\gamma_{n-1}, f_{n-1}^{(1 / m)}(x)$ and $e(1 / m) f_{0}^{(1 / m)}(x)$ are interchanged and other $f_{k}^{(1 / m)}(x)$ are unchanged.

From Lemma 4.2, a Schwarz map of (1.1) is given by

$$
z \in \boldsymbol{P}^{1}-\{0,1, \infty\} \longmapsto\left[f_{0}^{(1 / m)}(z): f_{1}^{(1 / m)}(z): \cdots: f_{n-1}^{(1 / m)}(z)\right] .
$$

We denote the closure of its image by $X_{n, p}^{(1 / m)}$, which is an irreducible curve in $\boldsymbol{P}^{n-1}$.

TheOrem 4.5. Assume $(p, q)=1$ and put $n=p+q, s=-p / n$ and $\alpha=$ $-1 /(m n), m \geq 2$. Then we have the equality

$$
\begin{aligned}
& X_{n, p}^{(1 / m)} \\
& =\left\{\left[y_{0}: y_{1}: \cdots: y_{n-1}\right] \in \boldsymbol{P}^{n-1} \mid \sigma_{k}\left(y_{0}^{m}, y_{1}^{m}, \ldots, y_{n-1}^{m}\right)=0,1 \leq k \leq n-1, k \neq q\right\},
\end{aligned}
$$

where $\sigma_{k}$ is the elementary symmetric function of degree $k$. Put

$$
\pi_{n, p}^{(1 / m)}\left(\left[y_{0}: y_{1}: \cdots: y_{n-1}\right]\right)=(-1)^{n} \frac{p^{p} q^{q}}{n^{n}} \frac{\left(\sigma_{q}\left(y_{0}^{m}, y_{1}^{m}, \ldots, y_{n-1}^{m}\right)\right)^{n}}{\left(\sigma_{n}\left(y_{0}^{m}, y_{1}^{m}, \ldots, y_{n-1}^{m}\right)\right)^{q}} .
$$

Then $z=\pi_{n, p}^{(1 / m)}(y)$ defines an $m^{n-1} n !: 1$ rational map of $X_{n, p}^{(1 / m)}$ to $\boldsymbol{P}^{1}$ satisfying

$$
\pi_{n, p}^{(1 / m)}\left(\left[f_{0}^{(1 / m)}(x): f_{1}^{(1 / m)}(x): \cdots: f_{n-1}^{(1 / m)}(x)\right]\right)=(-p)^{p} q^{q} n^{-n} x^{n} .
$$

The branch points of this map are $z=0,1, \infty$ with ramification indices $n, 2$, mpq, respectively.

Proof. We denote the right hand side of (4.3) by $\hat{X}_{n, p}^{(1 / m)}$ for the moment. Since

$$
\left(f_{j}^{(1 / m)}(x)\right)^{m}=f_{j}(x),
$$


we have, from Proposition 3.5, $X_{n, p}^{(1 / m)} \subset \hat{X}_{n, p}^{(1 / m)}$. By Bézout's theorem, $\pi_{n, p}^{(1 / m)}$ is an $m^{n-1} n !$ : 1 map of $\hat{X}_{n, p}^{(1 / m)}$ to $\boldsymbol{P}^{1}$ and from (3.8) it satisfies (4.5). On the other hand, $\pi_{n, p}^{(1 / m)}$ restricted to $X_{n, p}^{(1 / m)}$ has $m^{n-1} n$ ! points in any generic fiber because the covering transformation group of $X_{n, p}^{(1 / m)}$ includes $S_{n}$ from Corollary 4.4 and multiplication of $e(1 / m)$ to coordinate $y_{n-1}$ from Lemma 4.1. Hence we have $X_{n, p}^{(1 / m)}=\hat{X}_{n, p}^{(1 / m)}$. The ramification index at $z=\infty$ is $m p q$ from Proposition 2.6. This completes the proof.

COROLLARY 4.6. Let $\alpha=-1 /(m n), m \geq 2$, then the differential equation (1.1) has imprimitive finite irreducible projective monodromy group of order $m^{n-1} n$ !.

PROOF. The order of the projective monodromy group of (1.1) is equal to the degree of $\pi_{n, p}^{(1 / m)}$, which is $m^{n-1} n$ ! from the above theorem. Let $\Gamma_{0}$ and $\Gamma_{1}$ be loops once surrounding $z=0$ and $z=1$, respectively. From Lemma 4.1 and Corollary 4.4 , both $\Gamma_{0}$ and $\Gamma_{1}$ induce permutations on the set $\left\{\left\langle f_{j}^{(1 / m)}\right\rangle \mid 0 \leq j \leq n-1\right\}$ of one dimensional subspaces $\left\langle f_{j}^{(1 / m)}\right\rangle$. Hence the monodromy group of (1.1) is imprimitive.

Since neither $(-\alpha+k) / p-l / n$ nor $(\alpha+k) / q-l / n$ is an integer for any integers $k$ and $l,(1.1)$ is irreducible from Proposition 3.3 of $[\mathrm{B}-\mathrm{H}]$.

COROLLARY 4.7. For any positive integer $m, n$ and $q$ satisfying $1 \leq q \leq n-1$ and $(n, q)=1$, the algebraic set

$$
\left\{\left[y_{0}: y_{1}: \cdots: y_{n-1}\right] \in \boldsymbol{P}^{n-1} \mid \sigma_{k}\left(y_{0}^{m}, y_{1}^{m}, \ldots, y_{n-1}^{m}\right)=0,1 \leq k \leq n-1, k \neq q\right\}
$$

is irreducible.

PROOF. The statement is true for $m=1$ from Proposition 3.5 and for $m \geq 2$ from Theorem 4.4.

5. $\psi(\alpha,-1 / 3, x)$. In this section, we give several results concerning to $\psi(\alpha,-1 / 3, x)$.

5.1. A proof of Cardano's formula.

LEMMA 5.1.

$$
\begin{aligned}
& \psi(-1 / 2,-1 / 2, x)=\frac{-x+\sqrt{x^{2}+4}}{2} \\
& \psi(-1,1, x)=\frac{1+\sqrt{1-4 x}}{2}
\end{aligned}
$$

PROOF. From (2.17) and (2.18), we have

$$
\psi(-1 / 2,-1 / 2, x)={ }_{2} F_{1}\left(\frac{1}{2},-\frac{1}{2} ; \frac{1}{2} ;-\frac{1}{4} x^{2}\right)-\frac{1}{2} x_{2} F_{1}\left(1,0 ; \frac{3}{2} ;-\frac{1}{4} x^{2}\right) .
$$

Since ${ }_{2} F_{1}(a, b ; b ; x)=(1-x)^{-a},(5.1)$ is proved. 
If $k \geq 1$, then we have

$$
\begin{aligned}
c_{k}(-1,1) & =-(k, k-1) / k ! \\
& =-k(k+1) \cdots(2 k-2) / k !=-(2 k-2) ! /(k !(k-1) !) \\
& =-1 \cdot 3 \cdots(2 k-3) 2^{k-1} / k !=-(1 / 2, k-1) 2^{2 k-2} / k ! \\
& =(-1 / 2, k) 4^{k} /(2 k !) .
\end{aligned}
$$

Hence we have (5.2).

\section{LEMMA 5.2 .}

$$
\begin{gathered}
\psi(-1 / 3,-1 / 3, x) \\
=\left(\frac{1}{2}\left(1+\frac{4}{27} x^{3}\right)^{1 / 2}+\frac{1}{2}\right)^{1 / 3}-\frac{1}{3} x\left(\frac{1}{2}\left(1+\frac{4}{27} x^{3}\right)^{1 / 2}+\frac{1}{2}\right)^{-1 / 3} \\
=\left(\frac{1}{2}\left(1+\frac{4}{27} x^{3}\right)^{1 / 2}+\frac{1}{2}\right)^{1 / 3}-\left(\frac{1}{2}\left(1+\frac{4}{27} x^{3}\right)^{1 / 2}-\frac{1}{2}\right)^{1 / 3},
\end{gathered}
$$

where cube roots take positive values if $x$ is a positive small number.

ProOF. From (2.17) and (2.18), we have

$$
\begin{aligned}
& \psi(-1 / 3,-1 / 3, x) \\
& \quad={ }_{3} F_{2}\left(\frac{1}{3},-\frac{1}{6}, \frac{1}{3} ; \frac{2}{3}, \frac{1}{3} ;-\frac{4}{27} x^{3}\right)-\frac{1}{3} x_{3} F_{2}\left(\frac{2}{3}, \frac{1}{6}, \frac{2}{3} ; \frac{4}{3}, \frac{2}{3} ;-\frac{4}{27} x^{3}\right) \\
& ={ }_{2} F_{1}\left(-\frac{1}{6}, \frac{1}{3} ; \frac{2}{3} ;-\frac{4}{27} x^{3}\right)-\frac{1}{3} x_{2} F_{1}\left(\frac{1}{6}, \frac{2}{3} ; \frac{4}{3} ;-\frac{4}{27} x^{3}\right),
\end{aligned}
$$

which is equal to, from Remark 2.1,

$$
\begin{aligned}
\varphi_{0}\left(-1 / 3,1 / 1 ;-x^{3} / 27\right)-1 / 3 x \varphi_{0}\left(1 / 3,1 / 1 ;-x^{3} / 27\right) \\
=\psi\left(-1 / 3,1 ;-x^{3} / 27\right)-1 / 3 x \psi\left(1 / 3,1 ;-x^{3} / 27\right) \\
=\left[\psi\left(-1,1 ;-x^{3} / 27\right)\right]^{1 / 3}-1 / 3 x\left[\psi\left(-1,1 ;-x^{3} / 27\right)\right]^{-1 / 3} \\
=\left[\frac{1+\sqrt{1+4 x^{3} / 27}}{2}\right]^{1 / 3}-\frac{1}{3} x\left[\frac{1+\sqrt{1+4 x^{3} / 27}}{2}\right]^{-1 / 3}
\end{aligned}
$$

due to (5.2). This proves the lemma.

THEOREM 5.3 (Cardano). The equation

$$
X^{3}+3 p X-2 q=0
$$

has roots

$$
\varepsilon_{3}^{m}\left(q+\sqrt{p^{3}+q^{2}}\right)^{1 / 3}+\varepsilon_{3}^{2 m}\left(q-\sqrt{p^{3}+q^{2}}\right)^{1 / 3}, \quad 0 \leq m \leq 2,
$$

where $\varepsilon_{3}=e^{2 \pi i / 3}$ and cube roots must be chosen such that

$$
\left(q+\sqrt{p^{3}+q^{2}}\right)^{1 / 3}\left(q-\sqrt{p^{3}+q^{2}}\right)^{1 / 3}=-p .
$$


PROOF. Theorem follows from Lemma 5.2 and Proposition 2.6.

5.2. A uniformization of $\psi(-1 / 12,-1 / 3, x)$.

LEMMA 5.4. Let $s=-p / n$. Then for any $\alpha$, we have

$$
\prod_{j=0}^{n-1} \psi\left(\alpha, s, \varepsilon_{n}^{j} x\right)=1
$$

ProOF. From (2.19), we have

$$
\psi\left(\alpha, s, \varepsilon_{n}^{j} x\right)=\sum_{k=0}^{n-1} \varepsilon_{n}^{j k} \varphi_{k}(\alpha, s, x)
$$

First we note

$$
\varphi_{0}(0, s, x)=1, \quad \frac{\partial \varphi_{0}}{\partial \alpha}(0, s, x)=0 \quad \text { and } \quad \varphi_{k}(0, s, x)=0 \quad \text { for } k \geq 1 .
$$

Put $f(\alpha)=\prod_{j=0}^{n-1} \psi\left(\alpha, s, \varepsilon_{n}^{j} x\right)$. Then $f(0)=1$ and

$$
\begin{aligned}
\left.\frac{d f}{d \alpha}\right|_{\alpha=0} & =\left.\sum_{k=0}^{n-1} \frac{\partial \psi}{\partial \alpha}\left(\alpha, s, \varepsilon_{n}^{k} x\right) \prod_{j \neq k} \psi\left(\alpha, s, \varepsilon_{n}^{j} x\right)\right|_{\alpha=0}=\left.\sum_{k=0}^{n-1} \frac{\partial \psi}{\partial \alpha}\left(\alpha, s, \varepsilon_{n}^{k} x\right)\right|_{\alpha=0} \\
& =\left.\sum_{k=0}^{n-1} \sum_{j=0}^{n-1} \varepsilon_{n}^{j k} \frac{\partial \varphi_{j}}{\partial \alpha}\right|_{\alpha=0}=\left(\left.\sum_{j=1}^{n-1} \frac{\partial \varphi_{j}}{\partial \alpha}\right|_{\alpha=0}\right)\left(\sum_{k=0}^{n-1} \varepsilon_{n}^{j k}\right) \\
& =0 .
\end{aligned}
$$

Since $f(\alpha+\beta)=f(\alpha) f(\beta)$, we have $f(\alpha)=f(0) \exp (\alpha d f(0) / d \alpha)$. This proves (5.6).

Let $\alpha=-1 /(3 m)$ and put $y_{j}=f_{j}^{(1 / m)}(\alpha,-1 / 3, x)$ for $j=0,1,2$ (as for $f_{j}^{(1 / m)}$, see (4.1)). Then, from (4.3), (4.4) and (4.5), we have

$$
y_{0}^{m}+y_{1}^{m}+y_{2}^{m}=0, \quad \pi_{3,1}^{(1 / m)}\left(\left[y_{0}: y_{1}: y_{2}\right]\right)=\frac{\left(y_{0}^{2 m}+y_{1}^{2 m}+y_{2}^{2 m}\right)^{3}}{54\left(y_{0} y_{1} y_{2}\right)^{2 m}}=-\frac{4}{27} x^{3} .
$$

Let

$$
J(\tau)=12^{-3} h^{-2}\left(1+744 h^{2}+196884 h^{4}+21493760 h^{6}+\cdots\right), \quad h=e^{\pi i \tau}
$$

be the elliptic modular function defined on the upper half plane.

LEMMA 5.5. On the upper half plane $\{\tau \mid \operatorname{Im}(\tau)>0\}$, we have a single-valued function $x=x(\tau)$ so that $J(\tau)=-4 x^{3} / 27$ and that $x \geq 0$ for $\tau=e(1 / 3)+$ ti with $t \geq 0$.

Proof. The assertion holds because $J(\tau) \leq 0$ on the half line $\tau=e(1 / 3)+t i$ with $t \geq 0$ and because $J(\tau)$ has only triple zeros.

Now we have the following theorem. 
THEOREM 5.6. Let $m=4, n=3, p=1$ and $\alpha=-1 /(m n), s=-p / n$. Let $f_{j}^{(1 / m)}(x), j=0,1,2$ be solutions of (1.3) defined by (4.1). Let $x=x(\tau)$ be the singlevalued function in the previous lemma. Then we have

$$
\begin{aligned}
& f_{0}^{(1 / 4)}(x(\tau))=C \vartheta_{2}(0, \tau), \quad f_{1}^{(1 / 4)}(x(\tau))=C \vartheta_{0}(0, \tau), \\
& f_{2}^{(1 / 4)}(x(\tau))=e(1 / 8) C \vartheta_{3}(0, \tau),
\end{aligned}
$$

where $h=e^{\pi i \tau}, H_{0}=\prod_{k=1}^{\infty}\left(1-h^{2 k}\right)$ and $C=2^{-1 / 3} e(1 / 24) h^{-1 / 12} H_{0}^{-1}$.

Proof. Let $C_{4}=\left\{\left[y_{0}: y_{1}: y_{2}\right] \in \boldsymbol{P}^{2} \mid y_{0}^{4}+y_{1}^{4}+y_{2}^{4}=0\right\}$. Then

$$
\pi_{3,1}^{(1 / 4)}: C_{4} \rightarrow P^{1}
$$

satisfies, from (5.7),

$$
\pi_{3,1}^{(1 / 4)}\left(\left[y_{0}: y_{1}: y_{2}\right]\right)=\frac{\left(y_{0}^{8}+y_{1}^{8}+y_{2}^{8}\right)^{3}}{54\left(y_{0} y_{1} y_{2}\right)^{8}} .
$$

It is well-known (see, for example [Akh]) that

$$
\pi_{3,1}^{(1 / 4)}\left(\left[\vartheta_{2}(0, \tau): \vartheta_{0}(0, \tau): e(1 / 8) \vartheta_{3}(0, \tau)\right]\right)=J(\tau)
$$

This together with the equality (5.6) implies that both

$$
\left[f_{0}^{(1 / 4)}: f_{1}^{(1 / 4)}: f_{2}^{(1 / 4)}\right] \quad \text { and }\left[\vartheta_{2}(0, \tau): \vartheta_{0}(0, \tau): e(1 / 8) \vartheta_{3}(0, \tau)\right]
$$

belong to the same fiber $\left(\pi_{3,1}^{(1 / 4)}\right)^{-1}(J(\tau))$. Hence for some fourth roots $\varepsilon, \varepsilon^{\prime}$ of 1 and some function $C^{\prime}=C^{\prime}(\tau)$, we have

$$
\left\{f_{0}^{(1 / 4)}, f_{1}^{(1 / 4)}, f_{2}^{(1 / 4)}\right\}=\left\{C^{\prime} \vartheta_{2}(0, \tau), C^{\prime} \varepsilon \vartheta_{0}(0, \tau), C^{\prime} \varepsilon^{\prime} e(1 / 8) \vartheta_{3}(0, \tau)\right\}
$$

If we put $\tau=(-1+\sqrt{3} i) / 2+t i$ and let $t$ to $+\infty$, then $z=J(\tau)<0$ goes to $-\infty$. Since, from (5.3),

$$
\left.f_{j}^{(1 / 4)}=\varepsilon_{12}^{j} 2^{-1 / 12}((\sqrt{1-J(\tau)})+1)^{1 / 3}-\varepsilon_{3}^{j}(\sqrt{1-J(\tau)}-1)^{1 / 3}\right)^{1 / 4},
$$

we have (5.8) for some function $C=C(\tau)$ of $\tau$. Since $\vartheta_{2}(0, \tau) \vartheta_{0}(0, \tau) \vartheta_{3}(0, \tau)=2 h^{1 / 4} H_{0}^{3}$ ([Akh]), $C$ takes the value in the statement of the theorem.

REMARK 5.1. We dealt with the case of $m=4$ because we used the identity

$$
\vartheta_{0}^{4}(0, \tau)+\vartheta_{2}^{4}(0, \tau)-\vartheta_{3}^{4}(0, \tau)=0
$$

in the proof.

COROLLARY 5.7. Let a multi-valued function $f(z)$ be a solution of

$$
{ }_{3} E_{2}(1 / 12,-1 / 24,11 / 24 ; 1 / 3,2 / 3) \text {. }
$$

Then $f(J(\tau))$ turns out to be single-valued and a linear combination of $C \vartheta_{j}(0, \tau), j=$ $0,2,3$, where $C$ is as in Theorem 5.6. 


\section{REFERENCES}

[A-I] K. Аомото AND K. IGUChI, On quasi-hypergeometric functions, Methods Appl. Anal. 6 (1999), 55-66.

[Akh] N. I. AKHIEZER, Elements of the Theory of Elliptic Functions, Transl. Math. Monogr. 79, American Mathematical Society, Providence, RI, 1990.

[B-H] F. BeuKers AND G. Heckman, Monodromy for the hypergeometric function ${ }_{n} F_{n-1}$, Invent. Math. 95 (1989), 325-354.

[Blr] G. Belardinelli, Fonctions Hypergéométriques de Plusieurs Variables et Résolution Analytique des Équations Algébriques Générales, Mémor. Sci. Math., Fasc. 145, Gauthiers Villars, Paris, 1960.

[Bly] W. N. BAILEY, Generalized Hypergeometric Series, Cambridge Tracts in Mathematics and Mathematical Phisics No. 32, 1935.

[Brn] B. C. BERnd, Ramanujan's Notebooks Part I, Springer-Verlag, New York, 1985.

[Erd] A. ERDÉLYI (Editor), Higher transcendental functions, Vol. I, MacGraw Hill, New York, 1953.

[Kt] M. KATO, Schwarz maps of ${ }_{3} F_{2}$ with finite irreducible monodromy groups, Kyushu J. Math. 52 (1998), 475-495.

DEPARTMENT OF MATHEMATICS

COLLEGE OF EDUCATION

UNIVERSITY OF THE RYUKYUS

NISHIHARA-CHO, OKINAWA 903-0213

JAPAN

E-mail address: mkato@edu.u-ryukyu.ac.jp
DEPARTMENT OF MATHEMATICS

GRaduATE SCHOOL OF SCIENCE AND TeChNOLOGY

KOBE UNIVERSITY

ROKKO, KOBE 657-8501

JAPAN

E-mail address: noumi@math.sci.kobe-u.ac.jp 